\title{
ISOPARAMETRIC HYPERSURFACES AND METRICS OF CONSTANT SCALAR CURVATURE*
}

\author{
GUILLERMO HENRY ${ }^{\dagger}$ AND JIMMY PETEAN
}

\begin{abstract}
We showed the existence of non-radial solutions of the equation $\Delta u-\lambda u+\lambda u^{q}=0$ on the round sphere $S^{m}$, for $q<(m+2) /(m-2)$, and study the number of such solutions in terms of $\lambda$. We show that for any isoparametric hypersurface $M \subset S^{m}$ there are solutions such that $M$ is a regular level set (and the number of such solutions increases with $\lambda$ ). We also show similar results for isoparametric hypersurfaces in general Riemannian manifolds. These solutions give multiplicity results for metrics of constant scalar curvature on conformal classes of Riemannian products.
\end{abstract}

Key words. Yamabe equation, isoparametric hypersurfaces.

AMS subject classifications. $53 \mathrm{C} 21$.

1. Introduction. Given a Riemannian metric $g$ on a closed manifold $M^{n}$ we denote by $[g]$ the family of metrics conformal to $g$. The classical Yamabe problem consists of finding metrics of constant scalar curvature in $[g]$. To study this problem H. Yamabe considered [32] what we will now call the Yamabe constant of $[g]$ and denote by $Y(M,[g])$, the infimum of the (normalized) total scalar curvature functional restricted to $[g]$ :

$$
Y(M,[g])=\inf _{h \in[g]} \frac{\int_{M} s_{h} d^{2} v_{h}}{\operatorname{Vol}(M, h)^{\frac{n-2}{n}}} .
$$

Here $s_{h}$ and $d v o l_{h}$ denote the scalar curvature and volume element of $h$.

The critical points of the total scalar curvature functional restricted to $[g]$ are the metrics in $[g]$ which have constant scalar curvature. Yamabe attempted to prove the existence constant scalar curvature metrics in $[g]$ by showing that $Y(M,[g])$ is realized. His proof contained a mistake but his statement was eventually proved to be correct in a series of beautiful articles by N. Trudinger [30], T. Aubin [3] and R. Schoen [26]. It is not difficult to see that in $[g]$ there cannot exist two metrics of constant scalar curvatures of different signs. Moreover, if there is a metric $h \in[g]$ of constant non-positive scalar curvature then any other metric in $[g]$ of constant scalar curvature is of the form $k g$ for some $k \in \mathbb{R}_{>0}$. But if $Y(M,[g])>0$ the situation is much more interesting. For instance S. Brendle showed in [5] that in high dimensional spheres there are conformal classes of metrics (different form the conformal class of the round metric) for which the space of unit volume constant scalar curvature metrics is a non-compact family. Finding metrics of constant scalar curvature in a conformal class $[g]$ amounts to solving what is called the Yamabe equation for $(M, g)$ : if we let $p=p_{n}=2 n /(n-2)$ then $h=u^{p-2} g$ has constant scalar curvature $\lambda$ if and only if

$$
-a_{n} \Delta_{g} u+s_{g} u=\lambda u^{p-1}
$$

\footnotetext{
* Received January 27, 2012; accepted for publication September 12, 2012.

†Departamento de Matemática, FCEyN, Universidad de Buenos Aires, Ciudad Universitaria, Pab. I., C1428EHA, Buenos Aires, Argentina (ghenry@dm.uba.ar). G. Henry is supported by a postdoctoral fellowship of CONICET.

${ }^{\ddagger}$ CIMAT, A.P. 402, 36000, Guanajuato. Gto., México (jimmy@cimat.mx); and Departamento de Matemáticas, FCEyN, Universidad de Buenos Aires, Argentina (on leave). J. Petean is supported by grant 106923-F of CONACYT.
} 
where $a_{n}=4(n-1) /(n-2)$. Expressing any metric in $h \in[g]$ as $h=u^{p-2} g$ the total scalar curvature functional restricted to $[g]$ becomes

$$
u \mapsto \frac{\int_{M} a_{n}\|\nabla u\|^{2}+s_{g} u^{2} d v o l_{g}}{\|f\|_{p}^{2}}
$$

and the Yamabe equation is the Euler-Lagrange equation of this functional. To find all solutions of the Yamabe equation in a fixed conformal class of positive Yamabe constant is an extremely difficult problem. One does have uniqueness of solutions in the case of the conformal class of an Einstein metric by a classical result of M. Obata [22], and this covers a big family of interesting examples. The only non-trivial case which is completely understood is the case of cylinders $\left(S^{n} \times S^{1},\left[g_{0}^{n}+T^{2} d t^{2}\right]\right)$ : here (and in the rest of this article) $g_{0}^{n}$ denotes the round metric of sectional curvature 1 on the $n$-sphere, $d t^{2}$ is the metric of diameter 1 in the circle and $T \in \mathbb{R}_{>0}$. This case was studied by R. Schoen [27] and O. Kobayashi [14, 15]: in this case all solutions are constant along the spheres $S^{n}$ and the number of solutions increase with $T$.

The principal interest in this article is to study multiplicity results for solutions of the Yamabe equation on the products $\left(S^{n} \times S^{k},\left[g_{0}^{n}+T^{2} g_{0}^{k}\right]\right)$, for $n, k \geq 2$. This problem was already considered by the second author in [25], where it is studied the number of solutions which depend only on the first variable, and are radial (i.e. invariant by the canonical $S O(n)$-action which leaves the poles fixed). The same type of results had actually been obtained previously (and in a more complete way) by Q. Jin, Y. Y. Li and H. Xu [13, Theorem 1.3] in another context.

In this article we will show the existence of non-radial solutions of the equation. Since $2<p_{n+k}<p_{n}$ it is enough to study solutions of the subcritical Yamabe equation on $S^{n}$ :

$$
-\Delta_{g_{0}} u+\lambda u=\lambda u^{q}
$$

where $\lambda$ is a positive constant (related to the scalar curvature of the product manifold) and $1<q<p_{n}-1$. This equation has already been studied by several authors. Of particular interest to us are the articles [13] that we just mentioned and the article [4], where M-F.Bidaut-Veron and L. Veron show that the constant $u \equiv 1$ is the only solution of the equation if $\lambda \leq n /(q-1)$. We will actually make use of this result later.

To discuss our main results let us recall that if $(M, g)$ is a Riemannian manifold a smooth function $f: M \rightarrow \mathbb{R}$ is called an isoparametric function if there exists a continuous function $a$ and a smooth function $b$ such that

$$
\begin{gathered}
\|\nabla(f)\|^{2}=b \circ f \\
\Delta_{g}(f)=a \circ f .
\end{gathered}
$$

Regular level sets of isoparametric fuctions are called isoparametric hypersurfaces. The study of isoparametric hypersurfaces has a long history. E. Cartan [6] proved that in the case of space forms a hypersurface $M$ is isoparametric (according to the 
previous definition) if and only if it has constant principal curvatures. For instance, isoparametric hypersurfaces in Euclidean space $\mathbb{R}^{n}$ are just the canonical embeddings of $S^{n-1}, \mathbb{R}^{n-1}$ or $S^{k} \times \mathbb{R}^{n-k-1}$ as was shown by T. Levi-Civita [16] (in the threedimensional case) and B. Segre [28]. But the situation is much more interesting in the case of the round sphere. The simplest examples are the regular orbits of codimension one isometric actions on the sphere. These are called homogeneous and include many interesting examples; a classification of homogeneous isoparametric hypersurfaces in the sphere was given by W. Y. Hsiang and H. B- Lawson [10]. But there are families of examples of non-homogeneous isoparametric hypersurfaces. The first examples were found by H. Ozeki and M. Takeuchi [23, 24]. Although many results have been obtained towards a classification of isoparametric hypersurfaces in the spheres (see for instance the articles by T. Cecil, Q. S. Chi and G. Jensen [7], S. Immervoll [11] and R. Miyaoka [17]) a complete classification is still missing.

We will show that given any isoparametric hypersurface $S \subset S^{n}$ and any $k \geq 2$, for any positive $T$ such that

$$
\frac{1}{T}>\frac{6(n+5)(n+k-1)-n(n-1)}{k(k-1)},
$$

there exists a solution $u$ of the Yamabe equation on $\left(S^{n} \times S^{k}, g_{0}^{n}+T g_{0}^{k}\right)$ so that $S \times S^{k}$ is a level surface of $u$. Therefore to understand all solutions to the Yamabe equation in Riemannian products of spheres one needs to classify all isoparametric hypersurfaces of the sphere; and the previous comments then give us an idea of the complexity of the problem.

To state our results more precisely, given an isoparametric hypersurface $S \subset S^{n}$ we let $l$ be number of distinct principal curvatures of $S$. Then $l$ can only be 1,2 , 3,4 or 6 as follows from the work of H. F. Münzner [18, 19]. Moreover, there is an isoparametric function $f$ associated to $S$ (i.e. $S$ is a regular level set of $f$ ) which is the restriction to $S^{n}$ of a homogeneous polynomial $F$ in $\mathbb{R}^{n+1}$ of degree $l$. In this situation we will say that $S$ has degree $l$. Let $\lambda_{i, n}=-i(n+i-1)$ (these are the eigenvalues of the Laplace operator on $S^{n}$ ) and

$$
\lambda_{i, n}^{l, q}=\frac{-\lambda_{i l, n}}{q-1}
$$

Note that for $l, q, n$ fixed the sequence is increasing in $i$. We will prove

THEOREM 1.1. Let $S$ be an isoparametric hypersurface of $S^{n}$ of degree $l$. Then for each positive integer $i$ there exist at least $i$ solutions of equation (1) on $S^{n}$ for $\lambda \in\left(\lambda_{i, n}^{l, q}, \lambda_{i+1, n}^{l, q}\right]$ which are constant along $S$.

In terms of solutions of the Yamabe equation in $\left(S^{n} \times S^{k}, g_{0}^{n}+T g_{0}^{k}\right), \lambda$ corresponds to $\left(1 / a_{n+k}\right)(n(n-1)+(1 / T) k(k-1))$. To be more explicit about what the theorem is saying in terms of multiplicity results for metrics of constant scalar curvature let us first consider the case $n=k=3$. In $S^{3}$ there are two types of isoparametric hypersurfaces, corresponding to the canonical isometric actions of $S O(3)$ and of $S O(2) \times S O(2)$ : the first ones have $l=1$ and for the second ones $l=2$. Note that $a_{6}=5$ and $p_{6}=3$. Let $\mu_{i}=-i(i+2)$ be the eigenvalues of the Laplace operator on $S^{3}$ and let $T_{i}=6 /\left(-5 \mu_{i}-6\right)$ for $i \geq 1, T_{0}=1$. Then Theorem 1.1 translates to: 
Corollary 1.2. For $T \in\left[T_{i+1}, T_{i}\right)$ there are $i+[i / 2]$ non-isometric unit volume metrics of constant scalar curvature in $\left[g_{0}^{3}+T g_{0}^{3}\right]$.

The generic connected components of the level surfaces of the corresponding solutions of the Yamabe equation are diffeomorphic to either $S^{2} \times S^{3}$ or $S^{1} \times S^{1} \times S^{3}$.

Let us now consider the general case $n, k \geq 2$. One needs to count the number of known isoparametric hypersurfaces (to be more precise one should say families of isoparametric hypersurfaces: each isoparametric hypersurfaces has associated an isoparametric function $f$ and then the family of isoparametric hypersurfaces given as the regular level sets of $f$ ). For a positive integer $m$ let $j_{0}$ be the number of 2 's that appear in the factorization of $m+1$. If $j_{0}=4 l+d$ with $d=0,1,2,3$, define $\gamma(m)=8 l+2^{d}$. Also, for a positive integer $r$ let $\phi(r)$ be the number of integers $s$ such that $1 \leq s \leq r-1$ and $s \equiv 0,1,2,4 \bmod (8)$. And define $\beta(m)=$

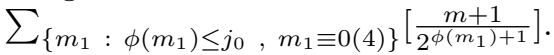

Let $\mu_{i}^{n, k}=(n+k-1) \lambda_{i, n}$ and $T_{i}^{n, k}=k(k-1) /\left(-\mu_{i}^{n, k}-n(n-1)\right)$, for $i \geq 1$. We have:

COROLlary 1.3. Let $N_{Y}^{n, k}(T)$ be the number of isometrically distinct unit volume metrics of constant scalar curvature in the conformal class of $\left(S^{n} \times S^{k}, g_{0}^{n}+T g_{0}^{k}\right)$ and suppose that $T \in\left[T_{i+1}^{n, k}, T_{i}^{n, k}\right)$. Then:

a) If $n=2 m$ with $m \neq 2$ then $N_{Y}^{n, k}(T) \geq i+[(2 m-1) / 2][i / 2]$.

b) If $n=2 m+1$ with $m \neq 1,3,4,6,7,9,12$ then $N_{Y}^{n, k}(T) \geq i+m[i / 2]+(\gamma(m)+$ $\beta(m))[i / 4]$.

c) 1. If $n=3$ then $N_{Y}^{n, k}(T) \geq i+[i / 2]$.

2. If $n=4$ then $N_{Y}^{n, k}(T) \geq i+[i / 2]+[i / 3]$.

3. If $n=7$ then $N_{Y}^{n, k}(T) \geq i+3[i / 2]+[i / 3]+[i / 4]+[i / 6]$.

4. If $n=9$ then $N_{Y}^{n, k}(T) \geq i+4[i / 2]+2[i / 4]$.

5. If $n=13$ then $N_{Y}^{n, k}(T) \geq i+6[i / 2]+[i / 3]+[i / 4]+[i / 6]$.

6. If $n=15$ then $N_{Y}^{n, k}(T) \geq i+7[i / 2]+4[i / 4]$.

7. If $n=19$ then $N_{Y}^{n, k}(T) \geq i+9[i / 2]+3[i / 4]$.

8. If $n=25$ then $N_{Y}^{n, k}(T) \geq i+12[i / 2]+[i / 3]+[i / 4]$.

Isoparametric hypersurfaces of degree 1 in $S^{n}$ are homogeneous, the orbits of the action of $O(n)$, the corresponding solutions to the Yamabe equation are the radial solutions discussed in $[13,25]$. These solutions account for all the $i$ 's in the corollary. Similarly isoparametric hypersurfaces of degree 2 are $S^{k} \times S^{n-k-1}$, the orbits of the isometric actions of $O(k+1) \times O(n-k)$, with $1 \leq k \leq[n-1 / 2]$ : these solutions account for all the $[i / 2]$ 's in the corollary. Isoparametric hypersurfaces of degree 3 were already classified by E. Cartan [6]: in this case the three principal curvatures have the same multiplicity which can be $1,2,4$ or 8 . The corresponding isoparametric hypersurfaces in $S^{4}, S^{7}, S^{13}$ and $S^{25}$ are called Cartan hypersurfaces and they are tubes over the canonical embeddings of the projective planes $\mathbf{F P}^{2}$ (where $\mathbf{F}$ are the real, complex, quaternionic or Cayley numbers). These account for all the $[i / 3]$ 's in the corollary. There are essentially two isoparametric hypersurfaces of degree 6 in the sphere, one in $S^{7}$ and the other in $S^{13}$. In both cases all the principal curvature have the same multiplicities, 1 and 2 respectively (see U. Abresch [1] and R. Miyaoka [17]). Isoparametric hypersurfaces of degree 4 provide the richest examples. The [i/4]'s appearing in the corollary come from a careful counting of all known isoparametric hypersurfaces of degree 4 . We give the details on this counting at the end of next 
section. It is known that new examples of isoparametric hypersurfaces of degree 4 can only appear in $S^{15}, S^{19}$ y $S^{31}$, see T. Cecil, Q. S. Chi and G. Jensen [7]. Therefore in all other dimensions we are counting all solutions of the Yamabe equation provided by Theorem 1.1.

For a general closed Riemannian manifold $(M, g)$ with an isoparametric hypersurface $S \subset M$ we will show in Section 3 that there is a subsequence of the sequence of eigenvalues of the Laplace operator on $(M, g)$ such that for each of the eigenvalues in the subsequence there exists an associated non-zero eigenfunction which is constant along $S$. Let us call $\lambda_{0}=\lambda_{0}(M, g, S)<0$ the first such eigenvalue.

TheOrem 1.4. Assume that $\left(M^{n}, g\right)$ is a closed Riemannian n-manifold of constant scalar curvature and $S$ an isoparametric hypersurface as above. If $\left(N^{k}, h\right)$ is a Riemannian $k$-manifold of constant scalar curvature let $\mathbf{s}=s_{g}+s_{h}$ be the scalar curvature of the product. If $\mathbf{s}>\frac{-a_{n+k} \lambda_{0}}{p_{n+k}-2}$ there is a smooth function $u: M \rightarrow \mathbb{R}$ which is constant along $S$ and solves the Yamabe equation for $(M \times N, g+h)$.

2. Some observations on isoparametric functions . Let $(M, g)$ be a closed connected Riemannian manifold and $f: M \rightarrow[a, b]$ a smooth surjective function. $f$ is called isoparametric if there exist a smooth function $b: \mathbb{R} \rightarrow \mathbb{R}$ and a continuous function $a: \mathbb{R} \rightarrow \mathbb{R}$ such that

$$
\|\nabla f\|^{2}=b(f)
$$

and

$$
\Delta f=a(f)
$$

Isoparametric functions have been studied for a long time, in particular by $\mathrm{E}$. Cartan [6] in space forms. We will mostly be interested in the case of the sphere but we will also use some results about isoparametric functions in general Riemannian manifolds: a good introduction to this general case is the work of Q. M. Wang [31].

If $f: M \rightarrow[a, b]$ is an isoparametric function then $a$ and $b$ are the only critical values of $f$. The level sets $f^{-1}(a)$ and $f^{-1}(b)$ are called the focal hypersurfaces of $f$. For $t \in(a, b)$, the regular level sets of $f, f^{-1}(t)$ are called isoparametric hypersurfaces.

We are going to be interested in the family of functions which are constant along the level surfaces of a fixed isoparametric function. We will use the following notation:

Definition 2.1. We call $S_{f}$ the family of functions on $S^{n}$ which are constant along level surfaces of $f$.

REMARK. We have not said if the functions on $S_{f}$ are meant to be continuous, or smooth, for instance. This is because we will need to use different function spaces. We will be explicit about the regularity assumed for functions in $S_{f}$ when needed.

The most familiar case is to consider an isometric codimension one action by a Lie group $G$ on $(M, g)$. Then any smooth function $f$ for which the level sets are the orbits of the action is isoparametric (equivalently, $f$ is the composition of the projection to the orbit space with an injective smooth function on the orbit space). Then $S_{f}$ is the family of $G$-invariant functions (or equivalently the family of functions obtained as the composition of $f$ with a function on the range of $f$ ). 
In the reminder of this section we will discuss the case of the round sphere. Therefore from now on let $f: S^{n} \rightarrow[a, b]$ be an isoparametric function on the sphere. Let $t$ be a regular value of $f$ and denote by $S_{t}=f^{-1}(t)$ the corresponding isoparametric hypersurface. It was shown by Cartan that $S_{t}$ has constant principal curvatures and this condition characterizes isoparametric hypersurfaces in space forms; but we will not make use of this fact.

It is clear that if $f$ is an isoparametric function and $u: \mathbb{R} \rightarrow \mathbb{R}$ is a smooth strictly monotone function then $u \circ f$ is also an isoparametric function. In the case of the sphere one has a certain normalization: if $M \subset S^{n}$ is an isoparametric hypersurface then Münzner proved in $[18,19]$ that there exists a homogeneous polynomial $F: \mathbb{R}^{n+1} \rightarrow \mathbb{R}$ of degree $l$ which satisfies what are known as the Cartan-Münzner equations:

$$
\begin{gathered}
<\nabla F, \nabla F>=l^{2}\|x\|^{2 l-2} \\
\Delta F=\frac{1}{2} c l^{2}\|x\|^{l-2},
\end{gathered}
$$

where $c$ is an integer which is given below, such that $M$ is a regular level set of $f=F_{S^{n}}$, which is an isoparametric function. Moreover, $l$ can only take the values $1,2,3,4$ or 6 and coincides with the number of distinct principal curvatures of $M$. In case $l=3$ (or 1 ) the principal curvatures have the same multiplicities and in case $l=2,4$ or 6 there are integers $m_{1}$ and $m_{2}$ (which might be equal) such that half of the principal curvatures have multiplicity $m_{1}$ and the other half $m_{2}$; in particular $(l / 2)\left(m_{1}+m_{2}\right)=n-1$. Then the constant $c$ is $m_{2}-m_{1}$. The polynomial $F$ is called a Cartan-Münzner polynomial. Note that interchanging $m_{1}$ and $m_{2}$ corresponds to replacing $F$ with $-F$. In this situation we will say that $f$ is an isoparametric function of degree $l$ and similarly that $M$ is an isoparametric hypersurface of degree $l$.

Recalling that the Laplace operator $\Delta_{S^{n}}$ on the sphere relates to the Laplacian on Euclidean space by the formula

$$
\Delta_{S^{n}}(u)=\left(\Delta U-\frac{\partial^{2} U}{\partial r^{2}}\right)-n \frac{\partial U}{\partial r}
$$

and

$$
\|\nabla(U)\|^{2}=\left(\frac{\partial U}{\partial r}\right)^{2}+\|\nabla(u)\|^{2}
$$

(where $r=\|x\|, U: \mathbb{R}^{n+1} \rightarrow \mathbb{R}$ and $u=\left.U\right|_{S^{n}}$ ) it is easy to check that

$$
\|\nabla(f)\|^{2}=b \circ f
$$

$$
\Delta_{S^{n}}(f)=a \circ f,
$$

$a(t)=-l(n+l-1) t+(1 / 2) c l^{2}, b(t)=-l^{2} t^{2}+l^{2}$. Note that since the only zeros of $b$ are \pm 1 it follows that the range of $f$ is $[-1,1]$. 
Let us see a few examples of isoparametric functions in spheres and the corresponding solution of the Cartan-Münzner equations.

EXAMPLE 2.2. The simplest example of a Cartan-Münzner polynomial, is given by $f(x)=f\left(x_{1}, \ldots, x_{n+1}\right)=x_{n+1}$. Then $\|\nabla f\|=\sqrt{1-x_{n+1}^{2}}=\sqrt{1-f^{2}}$ and $\Delta f=n x_{n+1}=n f . \quad f$ is invariant under the canonical $S O(n)$ action that fixes the poles and $S_{f}$ is just the family of radial functions.

EXAMPLE 2.3. Consider the obvious $O(n) \times O(k)$-action on $S^{n+k-1} \subset \mathbf{R}^{\mathbf{n}+\mathbf{k}}$. For $(x, y) \in \mathbf{R}^{\mathbf{n}+\mathbf{k}}$ write $x^{2}=x_{1}^{2}+\ldots+x_{n}^{2}$ and $y^{2}=y_{1}^{2}+\ldots+y_{k}^{2}$. Then $x^{2}$ and $y^{2}$ are homogeneous polynomials of degree 2 invariant under the action. Let $F=x^{2}-y^{2}$. Then $F$ is a Cartan-Münzner polynomial with $l=2, m_{1}=k-1, m_{2}=n-1$. The corresponding isoparametric hypersurfaces have two principal curvatures and are diffeomorphic to $S^{n-1} \times S^{k-1}$. As before we denote by $f=F_{\left.\right|_{S^{n}}}$; then $S_{f}$ is the family of $O(n) \times O(k)$-invariant functions.

EXAmple 2.4. If $z \in \mathbb{R}^{2 n+2}$ we write $z=(x, y) \in \mathbb{R}^{n+1} \times \mathbb{R}^{n+1}$. Consider the homogeneous polynomial $F: \mathbb{R}^{2 n+2} \rightarrow \mathbb{R}$ defined by $F(z)=\|z\|^{4}-2\left(\|x\|^{2}-\right.$ $\left.\|y\|^{2}\right)^{2}-8(\langle x, y\rangle)^{2}$. Then $F$ is a Cartan-Münzner polynomial with $l=4, m_{1}=1$, $m_{2}=n-1$. It is proved by K. Nomizu in [21] that the four distinct principal curvatures are $\frac{1+\sin (2 t)}{\cos (2 t)}, \frac{-1+\sin (2 t)}{\cos (2 t)}, \tan (t)$ and $-\cot (t)$ (the first two have multiplicity $n-1$ and the second two have multiplicity 1).

An isoparametric hypersurface $M$ of $S^{n}$ is homogeneous if there is a suitable subgroup of $O(n)$ that acts on $M$ transitively. These are the examples which are easier to understand. The previous examples are all homogeneous. And there exist much more examples, of course. All homogeneous isoparametric hypersurfaces have actually been classified by W. H. Hsiang and H. B. Lawson in [10]. But not all the isoparametric surfaces are homogeneous. The first examples were constructed by $\mathrm{H}$. Ozeki and M. Takeuchi in [23]:

EXAMPLE 2.5. Let $\mathbf{H}$ be the real quaternion algebra and let $u \longrightarrow \bar{u}$ be the natural involution. We can identify $\mathbb{R}^{16}$ with $\mathbf{H}^{2} \times \mathbf{H}^{2}$, we note $x \in \mathbb{R}^{16}$ as $x=\left(u_{0}, u_{1}, v_{0}, v_{1}\right)$ where $u_{i}, v_{i} \in \mathbf{H}$ for $i=\{0,1\}$. Let $F: \mathbb{R}^{16} \longrightarrow \mathbb{R}$ defined by

$$
F=\|x\|^{4}-2 F_{0}(x)
$$

where

$$
\begin{aligned}
F_{0}(x) & =4\left(u_{0} \bar{v}_{0}+u_{1} \bar{v}_{1}\right)\left(\bar{u}_{0} v_{0}+\bar{u}_{1} v_{1}\right) \\
& -\left(u_{0} \bar{v}_{0}+u_{1} \bar{v}_{1}+v_{0} \bar{u}_{0}+v_{1} \bar{u}_{1}\right)^{2} \\
& +\left[u_{1} \bar{u}_{1}-v_{1} \bar{v}_{1}+u_{0} \bar{v}_{0}+v_{0} \bar{u}_{0}\right]^{2}
\end{aligned}
$$

In [23] and [24], Ozeki and Takeuchi showed that the function F (that satisfied the equations (4) and (5) with $l=4$ and $c=1$ ) produced non homogeneous isoparametric hypersurfaces in $S^{15}$.

We now study the number of distinct isoparametric hypersurfaces of degree 4 which are known. This is the counting we used for Corollary 1.3. 
In the article [9] Ferus, Karcher and Münzner generalized the construction of Ozeki and Takeuchi to produce examples of homogeneous and non-homogeneous isoparametric hypersurfaces of degree 4: these are known as hypersurfaces of FerusKarcher-Münzner type or F-K-M hypersurfaces. The only known examples of isoparametric hypersurfaces of degree 4 which are not F-K-M are two homogeneous examples with multiplicities $(2,2)$ and $(4,5)$. S. Stolz [29] proved that the multiplicity of any isoparametric hypersurface of degree 4 is equal to one of these known examples. The multiplicities of F-K-M hypersurfaces are of the form $\left(m_{1}, m_{2}\right)=\left(m_{1}, l \delta\left(m_{1}\right)-m_{1}-1\right)$ where $\delta\left(m_{1}\right)$ is the unique positive integer such that the Clifford algebra of $m_{1}$ elements $C_{m_{1}-1}$ has an irreducible representation on $\mathbb{R}^{\delta\left(m_{1}\right)}$ and $l$ is an integer such that $m_{2}>0$, see T. Cecil, Q. S. Chi, G. R. Jensen [7]. It is known (see for instance [29]) that $\delta(r)=2^{\phi(r)}$ where $\phi(r)$ is the number of integers $s$ such that $1 \leq s \leq r-1$ and $s \equiv 0,1,2,4 \bmod (8)$. $\phi(r)$ is of course cyclic $\bmod (8)$. As a simplification for the reader, we present a table with the value of $\phi(r)$ for $1 \leq r \leq 16$ :

\begin{tabular}{||cc||cc||}
\hline$r$ & $\phi(r)$ & $r$ & $\phi(r)$ \\
\hline 1 & 0 & 9 & 4 \\
2 & 1 & 10 & 5 \\
3 & 2 & 11 & 6 \\
4 & 2 & 12 & 6 \\
5 & 3 & 13 & 7 \\
6 & 3 & 14 & 7 \\
7 & 3 & 15 & 7 \\
8 & 3 & 16 & 7 \\
\hline
\end{tabular}

The dimension of the hypersurface with multiplicities $\left(m_{1}, m_{2}\right)$ is of course $2\left(m_{1}+m_{2}\right)$. So there are no isoparametric hypersurfaces of degree 4 in evendimensional spheres. We want to count the number of such hypersurfaces in $S^{2 m+1}$. The homogeneous hypersurfaces with multiplicities $(2,2)$ and $(4,5)$ provide one example in $S^{9}$ and one in $S^{19}$. The case $m_{1}=1$ in the previous discussion provides one F-K-M hypersurface for any $m \geq 2$. Ferus, Karcher and Münzner proved in [9] that if $m_{1} \equiv 0 \bmod (4)$, there are $[l / 2]+1$ incongruent families of isoparametric hypersurfaces (two families are called congruent if the corresponding Cartan-Münzner polynomials are related by an isometry of the sphere) with multiplicity $\left(m_{1}, l \delta\left(m_{1}\right)-m_{1}-1\right)$. For all other $m_{1}$ 's there is only one F-K-M.

We provide the table with the pairs $\left(m_{1}, m_{2}\right)$ that correspond to families of isoparametric surfaces of F-K-M type with multiplicities $\left(m_{1}, m_{2}, m_{1}, m_{2}\right)$ for small values of $m_{1}$ and $m_{2}$ to simplify checking Corollary 1.3 (c):

\begin{tabular}{|c|l|}
\hline$(1, k-2)$ & $(1,1),(\mathbf{1}, \mathbf{2}),(1,3),(1,4),(\mathbf{1}, \mathbf{5}),(1,6),(1,7), \ldots$ \\
$(2,2 k-3)$ & $(\mathbf{2}, \mathbf{1}),(2,3),(\mathbf{2}, \mathbf{5}),(2,7),(2,9),(2,11),(2,13), \ldots$ \\
$(3,4 k-4)$ & $(\mathbf{3}, \mathbf{4}),(3,8),(3,12),(3,16),(3,20),(3,24), \ldots$ \\
$(4,4 k-4)$ & $(\mathbf{4}, \mathbf{3}),(4,7),(4,11),(4,15),(4,19),(4,23), \ldots$ \\
$(5,8 k-6)$ & $(\mathbf{5}, \mathbf{2}),(5,10),(5,18),(5,26),(5,34),(5,42), \ldots$ \\
$(6,8 k-7)$ & $(\mathbf{6}, \mathbf{1}),(\mathbf{6}, \mathbf{9}),(6,17),(6,25),(6,33),(6,41), \ldots$ \\
$(7,8 k-8)$ & $(\mathbf{7}, \mathbf{8}),(7,16),(7,24),(7,32),(7,40),(7,48), \ldots$ \\
$(8,8 k-9)$ & $(\mathbf{8}, \mathbf{7}),(8,15),(8,23),(8,31),(8,39),(8,47), \ldots$ \\
$(9,16 k-10)$ & $(\mathbf{9}, \mathbf{6}),(9,22),(9,38),(9,54),(9,70),(9,86), \ldots$ \\
$(10,32 k-11)$ & $(10,21),(10,53),(10,85),(10,107),(10,149), \ldots$ \\
$(11,64 k-12)$ & $(11,52),(11,116),(11,180),(11,244),(11,308), \ldots$ \\
$(12,64 k-13)$ & $(12,51),(12,115),(12,179),(12,243),(12,307), \ldots$ \\
$(13,128 k-14)$ & $(13,114),(13,242),(13,370),(13,498),(13,626), \ldots$ \\
$\vdots$ & $\ldots$
\end{tabular}


The family that correspond to $(2,1),(6,1),(5,2)$ and one of the family of $(4,3)$ (from the comments above we know that there are two of these) are congruent to those with multiplicities $(1,2),(1,6),(2,5)$ and $(3,4)$ respectively, and these are the only congruences among the hypersurfaces of F-K-M type, see T. Cecil [8] and Ferus, Karcher, Münzner [9]. So for instance for $2 m+1=7$ the pair $(1,2)$ provides the only F-K-M hypersurface in $S^{7}$. The $(1,3) \mathrm{F}-\mathrm{K}-\mathrm{M}$ and the homogeneous $(2,2)$ give the two examples in $S^{9}$. The $(1,4)$ and $(2,3) \mathrm{F}-\mathrm{K}-\mathrm{M}$ hypersurfaces provide the two examples in $S^{11}$. The $(1,5) \mathrm{F}-\mathrm{K}-\mathrm{M}$ hypersurface is the only example in $S^{13}$. In $S^{15}$ we have the F-K-M hypersurfaces of multiplicities $(1,6),(2,5),(3,4)$ and (the extra) $(4,3)$. In $S^{17}$ we only have the $(1,7)$ F-K-M hypersurface and in $S^{19}$ we have the homogeneous example with multiplicities $(4,5)$ and the F-K-M hypersurfaces of multiplicities $(1,8)$ and $(2,7)$.

Now assume that $m \geq 10$. If $m+1=2^{j} c$ and $m_{1}$ is such that $\delta\left(m_{1}\right)=2^{j}$ then we have an F-K-M hypersurface with multiplicities $\left(m_{1}, 2^{j} c-m_{1}-1\right)$ in $S^{2 m+1}$, as long as $m_{1}<\delta\left(m_{1}\right) c-1$ (but this condition is certainly verified if $m_{1}>8$ or $c>1$ and $m_{1} \neq 1$. For $c=1$ we would have $\delta\left(m_{1}\right)=m+1 \leq m_{1}+1 \leq 9$. Therefore we can forget about this condition when $m \geq 10)$. Then we have one F-K-M hypersurface for any $m_{1}$ such that $\phi\left(m_{1}\right) \leq j_{0}$. Therefore if we write $j_{0}=4 l+d$ with $l \geq 0$ and $d=0,1,2,3$ then there are $\gamma(m)=8 l+2^{d}$ F-K-M hypersurfaces in $S^{2 m+1}$.

Finally if $\phi\left(m_{1}\right) \leq j_{0}$ and $m_{1} \equiv 0 \bmod (4)$, we have $[l / 2]+1$ incongruent F-K-M hypersurfaces with $m_{2}=l \delta\left(m_{1}\right)-m_{1}-1$ (by the result in [9] mentioned above). We already counted one of them. Note that $l=(m+1) / 2^{\phi\left(m_{1}\right)}$. Then we call

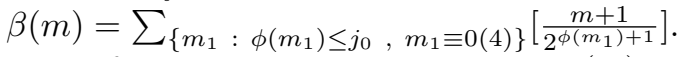

It follows that we have at least $\gamma(m)+\beta(m)$ different families of isoparametric hypersurface of degree 4 in $S^{2 m+1}$ with $m \geq 10$.

3. Eigenvalues of the Laplacian. Let $(M, g)$ be a closed Riemannian manifold and $f: M \rightarrow \mathbb{R}$ an isoparametric function. Then there exist eigenfunctions of the Laplace operator of $(M, g)$ which belong to $S_{f}$. Let us first observe the following:

LEMma 3.1. If $u \in S_{f}$ is smooth then $\Delta_{g} u \in S_{f}$.

Proof. Let $u=\varphi \circ f$, for a smooth function $\varphi$. Then by a direct computation $\Delta_{g} u=\varphi^{\prime \prime} b(f)+\varphi^{\prime} a(f) \in S_{f}$.

Proposition 3.2. There exist an infinite sequence $0<\lambda_{1}<\lambda_{2}<\lambda_{3}<\cdots<\infty$ such that there exists an eigenfunction $f_{i} \in S_{f}$ of $\Delta_{g}$ with eigenvalue $-\lambda_{i}$.

Proof. The constant function $1 \in S_{f}$ is an eigenfunction of the zero eigenvalue. We will use the usual arguments with Rayleigh quotients to characterize the following eigenfunctions with non zero eigenvalues in $S_{f}$. Let

$$
A_{1}=\left\{h \in S_{f} \cap H_{1}^{2}(M): \int h \operatorname{dvol}_{g}=0\right\}
$$

i.e. the functions in $S_{f} \cap H_{1}^{2}(M)$ orthogonal to the first eigenfunction ( $H_{1}^{2}$ means of course the Sobolev space with norm $\left.\|u\|_{1}^{2}=\|u\|_{2}+\|\nabla u\|_{2}\right)$. Consider

$$
\lambda_{1}=\inf _{h \in A_{1}-\{0\}} \frac{\int_{M}\|\nabla h\|^{2} d v o l_{g}}{\int_{M} h^{2} d v o l_{g}} .
$$

By the usual argument using the Rellich-Kondrakov theorem we can find a minimizing sequence $h_{i} \in A_{1}-\{0\}$ that converges to a minimizer $f_{1} \in H_{1}^{2}(M)$ with $\left\|f_{1}\right\|_{2}=1$, 
$\int f_{1}$ dvol $_{g}=0$ and $\left\|\nabla f_{1}\right\|_{2}^{2}=\lambda_{1}$. Since $A_{1}$ is a closed subspace of $H_{1}^{2}(M)$ it follows that $f_{1} \in A_{1}-\{0\}$ is a minimizer and so a critical point of the functional in $A_{1}-\{0\}$. This means that for all $u \in A_{1}$,

$$
\int_{M}\left(\Delta_{g} f_{1}+\lambda_{1} f_{1}\right) u d v o l_{g}=0
$$

and since $\Delta_{g} f_{1}+\lambda_{1} f_{1} \in A_{1}$ by Lemma 3.1, it follows that $f_{1}$ is eigenfunction of $\Delta_{g}$ with eigenvalue $-\lambda_{1}$. Then one defines $\left.A_{2}=A_{1} \cap<f_{1}\right\rangle^{\perp}$ and repeating the argument obtains and eigenfunction $f_{2}$ and so on.

The sequence $0>-\lambda_{1}>-\lambda_{2}>\ldots$ is of course a subsequence of the spectrum of $\Delta_{g}$. One can also see that:

Proposition 3.3. For each eigenvalue $\lambda_{i}$ the space of $S_{f}$-eigenfunctions has dimension one.

This is an easy consequence of the fact that $S_{f}$-eigenfunctions are obtained as solutions of a second order ordinary differential equation. We will do it again in the case of the round sphere, which is our main interest, so we omit the simple proof here.

In the case of an isoparametric function on the round sphere one can be much more precise. We look for eigenfunctions of the Laplace operator on $\left(S^{n}, g_{0}\right)$ which belong to $S_{f}$ for some isoparametric function $f$. Recall that the eigenvalues of $\Delta_{S^{n}}$ are $\lambda_{i, n}=-i(n+i-1) . f$ is an eigenfunction with eigenvalues $\lambda_{i}$ if and only if $f$ is the restriction to the sphere of a harmonic homogeneous polynomial in $\mathbb{R}^{n+1}$ of degree $i$.

LEMMA 3.4. Let $f: S^{n} \rightarrow[-1,1]$ be an isoparametric function obtained as the restriction to $S^{n}$ of a solution $F$ of de Cartan-Munzner equations. Let $l$ be the degree of $F$. Then for each $i=0,1, \ldots$ there is an eigenfunction $f_{i} \in S_{f}$ of the Laplacian $\Delta_{S^{n}}$ with eigenvalue $\lambda_{i l, n}$. The space of such eigenfunctions has dimension 1 and is generated by $p_{i} \circ f$ where $p_{i}$ is a monic polynomial of degree $i$ which has $i$ distinct simple roots in the interval $(-1,1)$. Moreover, if $\lambda_{j, n}$ is an eigenvalue of $\left.\Delta_{S^{n}}\right|_{S_{f}}$ then $j=i l$ for some $i$.

Proof. $F$ is a homogeneous polynomial of degree $l$ in $\mathbb{R}^{n+1}$ which solves the equations (4) and (5).

If $l$ is odd then $c=m_{2}-m_{1}=0$ and we let $U=F$. If $c \neq 0$ we let $U=$ $F-(c /(n+1))\left(x_{1}^{2}+\ldots+x_{n+1}^{2}\right)^{l / 2}$. Then $U$ is a harmonic homogeneous polynomial of degree $l$. Let $u=\left.U\right|_{S^{n}}$. Then $u \in S_{f}$ is an eigenfunction of $\Delta_{S^{n}}$ with eigenvalue $\lambda_{l, n}$. And of course $u=p_{1}(f)$ with $p_{1}(t)=t-(c /(n+1))$. Then $u$ verifies $S_{u}=S_{f}$, $\Delta u=\lambda_{l, n} u,\|\nabla u\|^{2}=\|\nabla f\|^{2}=l^{2}\left(1-f^{2}\right)=l^{2}\left(1-(u+c /(n+1))^{2}\right)$.

Now a function $\alpha \circ f$ is eingenfunction of $\Delta_{S^{n}}$ with eigenvalue $\lambda_{i, n}$ if and only if $\alpha$ solves the second order ordinary differential equation

$$
O_{i}(\alpha)=l^{2} \alpha^{\prime \prime}(t)\left(1-t^{2}\right)+\alpha^{\prime}(t)\left((1 / 2) c l^{2}-l(n+l-1) t\right)-\lambda_{i, n} \alpha(t)=0 .
$$

By a straightforward computation

$$
\begin{aligned}
O_{i l}\left(t^{i}\right) & =l^{2} i(i-1) t^{i-2}\left(1-t^{2}\right)+i t^{i-1}\left((1 / 2) c l^{2}-l(n+l-1) t\right)+i l(n+i l-1) t^{i} \\
& =C t^{i-1}+D t^{i-2}
\end{aligned}
$$


for some $C, D \in \mathbb{R}$. Moreover for $j<i O_{i l}\left(t^{j}\right)=E t^{j}+F t^{j-1}+G t^{j-2}$ for some $E, F, G \in \mathbb{R}$ and $E \neq 0$. We set $p_{0}=1, p_{1}(t)=t-(c /(n+1))$ (as before) and it follows that there is exactly one monic polynomial of degree $i, p_{i}$ which solves $O_{i l}\left(p_{i}\right)=0$. The fact that the roots of $p_{i}$ are simple is a simple consequence of the fact that $p_{i}$ is a non-trivial solution of a second order ordinary differential equation. The fact that it has $i$ roots in $(-1,1)$ is clear for $i=0$ and $i=1$. Assume that it is true for some $i$. Let $-1<t_{1}<\ldots<t_{i}<1$ be the $i$ roots. If we call $t_{0}=-1$ and $t_{i+1}=1$ then it is enough to prove that $p_{i+1}$ has at least one root in each interval $\left(t_{j}, t_{j+1}\right)$. This is a classical application of Sturm comparison theorem [12, page 229]: we have

$$
\begin{gathered}
p_{i}^{\prime \prime}+\frac{\left((1 / 2) c l^{2}-l(n+l-1) t\right)}{l^{2}\left(1-t^{2}\right)} p_{i}^{\prime}-\frac{\lambda_{i l, n}}{l^{2}\left(1-t^{2}\right)} p_{i}=0, \\
p_{i+1}^{\prime \prime}+\frac{\left((1 / 2) c l^{2}-l(n+l-1) t\right)}{l^{2}\left(1-t^{2}\right)} p_{i+1}^{\prime}-\frac{\lambda_{(i+1) l, n}}{l^{2}\left(1-t^{2}\right)} p_{i+1}=0,
\end{gathered}
$$

with $-\lambda_{(i+1) l, n}>-\lambda_{i l, n}$. Also

$$
\frac{p_{i+1}^{\prime}(-1)}{p_{i+1}(-1)}=\frac{\lambda_{(i+1) l}}{\left((1 / 2) c l^{2}+l(n+l-1)\right)}<\frac{\lambda_{i l}}{\left((1 / 2) c l^{2}+l(n+l-1)\right)}=\frac{p_{i}^{\prime}(-1)}{p_{i}(-1)} .
$$

Then if $p_{i+1}$ did not have a 0 between $-1=t_{0}$ and $t_{1}$ we would apply Sturm's Theorem to reach a contradiction. In a similar way one checks that $p_{i+1}$ must have a zero in each of the other intervals $\left(t_{j}, t_{j+1}\right)$; and this completes the proof that $p_{i}$ has exactly $i$ zeros in the interval $(-1,1)$.

To prove the last statement in the lemma, pick $j, i l<j<(i+1) l$. Let $\varphi$ be a non-trivial solution of $O_{j}(\varphi)=0$. Then applying the same Sturm comparison to $p_{i}$ and $\varphi$ would prove that $\varphi$ has at least $i+1$ zeros. And then applying the same Sturm comparison to $\varphi$ and $p_{i+1}$ would prove that $p_{i+1}$ has at least $i+2$ zeros, which is of course false.

4. Proof of Theorem 1.1. We will use bifurcation theory to prove Theorem 1.1. We will use the notation in [20]. This is the same analysis carried out in [13] to study solutions which are radial with respect to some axis, but we will see that it works in this more general case. The proof is based on Krasnoselski's Theorem and the global extension by P. Rabinowitz; this corresponds with sections 3.3 and 3.4 of [20].

To begin with let us recall the following

Definition 4.1. Given a Banach space $X$ and a $C^{r}$ map $H: X \times \mathbb{R} \rightarrow X a$ point $\left(0, \lambda_{0}\right)$ such that $H\left(0, \lambda_{0}\right)=0$ is called a bifurcation point if every neighborhood of $\left(0, \lambda_{0}\right)$ contains points $(x, \lambda)$ with $x \neq 0$ such that $H(x, \lambda)=0$.

Our first task is to write our equation as an operator equation as in bifurcation theory. Fix $q, 1<q<p_{n}-1$. Given a positive solution $u: S^{n} \rightarrow \mathbb{R}_{>0}$ of equation (1)

$$
-\Delta u+\lambda u=\lambda u^{q}
$$

we let $v=u-1$; then $v>-1$ and it is a solution of $E q_{\lambda}$ :

$$
-\Delta v=\lambda\left((v+1)^{q}-(v+1)\right) .
$$


Now fix an isoparametric function $f: S^{n} \rightarrow[-1,1]$, where $f=\left.F\right|_{S^{n}}$ for a homogeneous polynomial $F$ of degree $l$ satisfying the Cartan-Münzner equations. And consider the Banach space

$$
C^{2, \alpha}\left(S_{f}\right)=C^{2, \alpha}\left(S^{n}\right) \cap S_{f}
$$

Let $T: C^{2, \alpha}\left(S_{f}\right) \rightarrow C^{2, \alpha}\left(S_{f}\right)$ be the inverse of $-\Delta+I d: C^{4, \alpha}\left(S_{f}\right) \rightarrow C^{2, \alpha}\left(S_{f}\right)$; $T$ is a linear compact map. Consider the region

$$
A=\left\{(v, \mu) \in C^{2, \alpha}\left(S_{f}\right) \times \mathbb{R}: \mu>1, v>-1\right\},
$$

relate $\lambda$ and $\mu$ by the equation $\mu=(q-1) \lambda+1$ and define $g: A \rightarrow C^{2, \alpha}\left(S_{f}\right)$ by

$$
g(v, \mu)=\lambda T\left((v+1)^{q}-q v-1\right) .
$$

Then $g$ is a non-linear compact map. Then define $H: A \rightarrow C^{2, \alpha}\left(S_{f}\right)$ by

$$
H(v, \mu)=v-\mu T(v)-g(v, \mu) .
$$

Then $v$ is a solution of $E q_{\lambda}$ if and only if $H(v, \mu)=0$ (simply apply $-\Delta+I d$ to this equation). Of course one always has the trivial solution $v=0$, for any $\mu$. Moreover, $H$ satisfies the conditions to apply Krasnoselski's Theorem: $g(v, \mu)=o(\|v\|)$ uniformly on $|\mu|<\epsilon$ (for any constant $\epsilon$ ). Then one notes that $1 / \alpha$ is an eigenvalue of $T$ if and only if $1-\alpha$ is an eigenvalue of $\left.\Delta\right|_{S_{f}}$, with the same eigenspaces. Therefore it follows from Lemma 3.4 and Krasnoselski's Theorem [20, Theorem 3.3.1] that the points $\left(0, \mu_{i}\right)$ with $\mu_{i}=1+i l(n+i l-1)$ are bifurcation points (and the only ones).

To prove Theorem 1.1 we apply Rabinowitz' Theorem [20, Theorem 3.4.1].

We will prove

$\left.{ }^{*}\right)$ If $C$ is the closure of the non-trivial $(v \neq 0)$ solutions of $H(v, \mu)=0$ and $C_{i}$ is the connected component of $C$ containing $\left(0, \mu_{i}\right)$ then $C_{i}$ does not contain any other of the bifurcation points.

Assuming (*) it follows from Rabinowitz' Theorem that $C_{i}$ is not compact in $A$. In order to finish the proof of Theorem 1.1 it is enough to show that for any $\mu>\mu_{i}$ there exists $v \neq 0$ such that $(v, \mu) \in C_{i}$. If this were not the case then $C_{i}$ would be contained in a region of the form $A \cap\left(C^{2, \alpha}\left(S_{f}\right) \times[n /(q-1), D]\right)$ (there are no non-trivial solutions of $E q_{\lambda}$ for $\lambda \leq n /(q-1)$, [4, Theorem 6.1] ). But for uniformly bounded $\mu$, for all solutions of $\bar{H}(v, \mu)=0, v$ is uniformly bounded above and bounded below away from -1 by [13, Lemma 2.4 (b)]. This would imply that $C_{i}$ is compact and the proof follows.

To prove $(*)$ first note that the bifurcation points are the only points in $C$ with $v=0$. Next for any $v \in S_{f}$ let $z(v)$ be the number of zeros of $\varphi$ where $v=\varphi \circ f$ (it might be $\infty)$. Then for each positive integer $k$ consider the set $B_{k}=\left\{(v, \mu) \in S_{f}\right.$ : $H(v, \mu)=0, z(v)=k\}$. Note that if $H(v, \mu)=0$ then $u=v+1=\varphi \circ f$ where $\varphi$ solves the ordinary differential equation (where $\lambda=\lambda(\mu)$ )

$$
-\left(l^{2} \varphi^{\prime \prime}(t)\left(1-t^{2}\right)+\varphi^{\prime}(t)\left((1 / 2) c l^{2}-l(n+l-1) t\right)\right)+\lambda \varphi=\lambda \varphi^{q} .
$$


If $v \neq 0$ then $u \neq 1$ and $u$ must have a minimum which must be $<1$ and a maximum $>1$ and therefore it must take the value 1 a positive finite number of times with non-zero derivative. This implies: $k$.

i) All non-trivial solutions of $H(v, \mu)=0$ belong to $B_{k}$ for some positive integer

ii) Each $B_{k}$ is open in $C$.

Also by the local bifurcation theorem of Crandall and Rabinowitz [20, Theorem 3.2.2] the non-trival solutions near the bifurcation point $\left(0, \mu_{k}\right)$ can be parametrized by $s$, $0<|s|<\varepsilon$, by $\left(s p_{k}+o\left(s^{2}\right), \lambda(s)\right)$, which implies by Lemma 3.4 that non-trivial solutions near $\left(0, \mu_{k}\right)$ belong to $B_{k}$. Then define $C_{i}^{0}=\left(C_{i} \cap B_{i}\right) \cup\left\{\left(0, \mu_{i}\right)\right\}$. If we prove that $C_{i}^{0}=C_{i}$ we are done; and to prove this it is enough to show that $C_{i}^{0}$ is open and closed in $C_{i}$, but this follows easily from the previous lines.

We have therefore completed the proof of Theorem 1.1.

5. Proof of Theorem 1.4. Let $(M, g)$ and $(N, h)$ be closed Riemannian manifolds of constant scalar curvature with $\operatorname{dim} M=n$ and $\operatorname{dim} N=k$. Let us denote with $\mathbf{s}=s_{g}+s_{h}$ the scalar curvature of $(M \times N, g+h)$. Let $f: M \rightarrow \mathbb{R}$ be an isoparametric function such that the isoparametric hypersurface $S$ is a regular level set of $f$. Let $V=\operatorname{Vol}(N, h)$ and consider the Yamabe functional on $(M \times N, g+h)$ and restrict it to $S_{f}$ :

$$
Y(u)=\frac{a_{n+k}\|\nabla u\|_{2}^{2}+\mathbf{s}\|u\|_{2}^{2}}{\|u\|_{p}^{2}} V^{p-2 / p}=\frac{Q(u)}{\|u\|_{p}^{2}} V^{p-2 / p}
$$

with $2<p=p_{n+k}<p_{n}$. If $u, v \in S_{f}$ then

$$
\frac{d Y(u+t v)}{d t}(0)=\frac{2 V^{p-2 / p}}{\|u\|_{p}^{2}} \int\left(-a_{n+k} \Delta_{g} u+\mathbf{s} u-\|u\|_{p}^{-p} u^{p-1} Q(u)\right) v d v o l_{g}
$$

It follows from Lemma 3.1 that $u \in S_{f}$ is a critical point of $\left.Y\right|_{S_{f}}$ if and only if $u$ if a solution of the Yamabe equation $-a_{n+k} \Delta_{g} u+\mathbf{s} u=\beta u^{p-1}$ with $\beta=\|u\|_{p}^{-p+2} Y(u)$. From now on we write $a_{n+k}$ as $a$.

LEMma 5.1. There is a smooth positive function $u \in S_{f}$ which minimizes the Yamabe functional restricted to $S_{f}$.

Proof. Let $u_{i} \in H_{1}^{2}(M) \cap S_{f}$ be a sequence of smooth non-negative functions such that

$$
\lim _{i \rightarrow \infty} Y\left(u_{i}\right)=\inf _{v \in S_{f}} Y(v)=\alpha .
$$

Assume $\left\|u_{i}\right\|_{p}=1$. Since $p>2$ and $p<p_{n}$ one sees that the sequence $u_{i}$ is a bounded sequence in $H_{1}^{2}$ and apply the Rellich-Kondrakov theorem to see that there is a subsequence of $u_{i}$ that converges in $L_{p}$ to a function $u$. Then necessarily $u$ is a smooth positive solution of the Yamabe equation. This was actually carried out in [2, Proposition 2.2]. But $u \in S_{f}$ because $H_{1}^{2}(M) \cap S_{f}$ is a closed subset of $H_{1}^{2}(M)$.

LEMMA 5.2. Assume that there is a non-constant eigenfunction of the Laplacian $w \in S_{f}$ with $\Delta w=\mu w$. If $\mathbf{s}>\frac{-a \mu}{p-2}$ then the constant function is not the minimizer of the Yamabe functional restricted to $S_{f}$. 
Proof. Let $y(t)=Y(1+t w)$. Then $y^{\prime}(0)=0$ (this is essentially the fact that $g+h$ has constant scalar curvature). And

$$
\frac{d^{2} y}{d t^{2}}(0)=2(\operatorname{Vol}(M \times N, g+h))^{-\frac{2}{p}}[(2-p) \mathbf{s}-\mu . a] \int w^{2} d v o l_{g}
$$

It follows from the hypothesis that the expression above is negative, which proves the Lemma. $\mathrm{\square}$

Proof of Theorem 1.4. We are considering an isoparametric function $f: M \longrightarrow \mathbb{R}$ such that $S$ is one of its regular level sets and $\lambda_{0}<0$ is the first negative eigenvalue of $\left.\Delta_{g}\right|_{S_{f}}$ (see Proposition 3.2). Then the previous Lemma tells us that (under the conditions of Theorem 1.4) the constant functions do not minimize the Yamabe functional restricted to $S_{f}$. Hence Lemma 5.1 says that there exists a positive nonconstant function $u \in S_{f}$ which minimizes $Y$ in $S_{f}$. Therefore $u$, seen as a function from $M \times N$ to $\mathbb{R}_{>0}$, is a solution of the Yamabe equation in $(M \times N, g+h)$ and $S$ is included in a level set of $u$.

\section{REFERENCES}

[1] U. ABResch, Isoparametric hypersurfaces with four or six distinct principal curvatures, Math. Ann, 264 (1983), pp. 283-302.

[2] K. Akutagawa, L. Florit, and J. Petean, On Yamabe constants of Riemannian products, Comm. Anal. Geom., 15 (2007), pp. 947-969.

[3] T. Aubin, Equations differentielles non-lineaires et probleme de Yamabe concernant la courbure scalaire, J. Math. Pures Appl., 55 (1976), pp. 269-296.

[4] M.-F. Bidaut-Veron and L. Veron, Nonlinear elliptic equations on compact Riemannian manifolds and asymptotics of Emden equations, Invent. math., 106 (1991), pp. 489-539.

[5] S. Brendle, Blow-up phenomena for the Yamabe equation, Journal Amer. Math. Soc., 21 (2008), pp. 951-979.

[6] E. Cartan, Familles de surfaces isoperimetriques dans les espaces a courbure constante, Ann. Mat. Pura Appl., 17 (1938), pp. 177-191.

[7] T. CECIL, Q. S. ChI, AND G. JENSEN, Isoparametric hypersurfaces with four different principal curvatures, Ann. Math., 166 (2007), pp. 1-76.

[8] T. CECIL, Isoparametric and Dupin hypersurfaces, Symmetry, Integrability and Geometry: Methods and Applications, SIGMA, 4 (2008), 062, pp. 1-28.

[9] D. Ferus, H. Karcher, and H. F. Münzner, Cliffordalgebren und neue isoparametrische Hyperflachen, Math. Z., 177 (1981), pp. 479-502.

[10] W. Y. Hsiang And H. B. Lawson, Minimal submanifolds of low cohomogeneity, J. Differential Geometry, 5 (1971), pp. 1-38.

[11] S. ImmervolL, The classification of isoparametric hypersurfaces with four distinct principal curvatures, Ann. Math., 168 (2008), pp. 1011-1024.

[12] E. L. Ince, Ordinary Differential Equations, Dover Publications, New York, 1956.

[13] Q. Jin, Y. Y. LI, AND H. XU, Symmetry and asymmetry: the method of moving spheres, Advances in Differential Equations, 13 (2008), pp. 601-640.

[14] O. Kobayashi, On the large scalar curvature, Research Report 11, Dept. Math. Keio Univ. (1985).

[15] O. KobaYAShi, Scalar curvature of a metric with unit volume, Math. Ann, 279 (1987), pp. 253265.

[16] T. Levi-Civita, Famiglie di superficie isoparametrische nell'ordinario spacio euclideo, Atti. Accad. naz. Lincei. Rend. Cl. Sci. Fis. Mat. Natur, 26 (1937), pp. 355-362.

[17] R. MiYaoka, The Dorfmeister-Neher theorem on isoparametric hypersurfaces, Osaka J. Math., 46 (2009), pp. 695-715.

[18] H. F. MüNZnER, Isoparametrische Hyperflachen in spharen I, Math. Ann., 251 (1980), pp. 5771.

[19] H. F. MünZnER, Isoparametrische Hyperflachen in spharen II, Math. Ann., 256 (1981), pp. 215232. 
[20] L. NiRenBerG, Topics in nonlinear functional analysis, New York University Lecture Notes, New York, 1974.

[21] K. Nomizu, Some results in E. Cartan's theory of isoparametric families of hypersurfaces, Bulletin of the American Mathematical Society, 79 (1973), pp. 1184-1188.

[22] М. Овата, The conjectures on conformal transformations of Riemannian manifolds, J. Diff. Geom., 6 (1971), pp. 247-258.

[23] H. OZEKI AND M. TAKEUCHI, On some types of isoparametrics hypersurfeces in sphere I, Tohoku Math. Journ., 27 (1975), pp. 515-559.

[24] H. OzeKi And M. TAKeuchi, On some types of isoparametrics hypersurfeces in sphere II, Tohoku Math. Journ., 28 (1976), pp. 7-55.

[25] J. Petean, Metrics of constant scalar curvature conformal to Riemannian products, Proceedings of the American Mathematical Society, 138 (2010), pp. 2897-2905.

[26] R. Schoen, Conformal deformation of a Riemannian metric to constant scalar curvature, J. Differential Geometry, 20 (1984), pp. 479-495.

[27] R. Schoen, Variational theory for the total scalar curvature functional for Riemannian metrics and related topics, Lectures Notes in Math. 1365, Springer-Verlag, Berlin, 1989, pp. 120 154 .

[28] B. SEgRe, Famiglie di ipersuperficie superficie isoparametrische negli spazi euclidei ad un qualunque numero di demesioni, Atti. Accad. naz. Lincei. Rend. Cl. Sci. Fis. Mat. Natur, 27 (1938), pp. 203-207.

[29] S. Stolz, Multiplicities of Dupin Hypersurfaces, Invent. Math., 138 (1999), pp. 253-279.

[30] N. TRUdinger, Remarks concerning the conformal deformation of Riemannian structures on compact manifolds, Ann. Scuola Norm. Sup. Pisa, 22 (1968), pp. 265-274.

[31] Q. M. WANG, Isoparametric functions on Riemannian Manifolds. I, Math. Ann., 277 (1987), pp. 639-646.

[32] H. YAMABE, On a deformation of Riemannian structures on compact manifolds, Osaka Math. J., 12 (1960), pp. 21-37. 
G. HENRY AND J. PETEAN 